\title{
Post-Operative Pain Management in Patients Undergoing Robotic Urological Surgery
}

\author{
Sian E. Batley ${ }^{a} \quad$ Venkat Prasad ${ }^{a} \quad$ Nikhil Vasdev $^{b} \quad$ Gowrie Mohan-S $S^{a}$

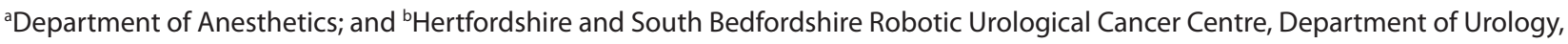 \\ Lister Hospital, Stevenage, UK
}

\section{Key Words}

Robotic surgery • Pain management • Enhanced recovery

\begin{abstract}
Robotic urological surgery is being increasingly performed worldwide. The main focus currently is on the operative technique but post operative patient care is an essential part of the process to make this technique safe and successful. We present a review on multiple analgesic techniques available to prevent and treat pain specifically caused after by urological robotic surgery; this article will explain the mechanism of pain pathways involved in laparoscopic procedures and review current evidence pertaining to systemic and regional analgesia methods.

Copyright $\odot 2015$ S. Karger AG, Basel
\end{abstract}

\section{Introduction}

Robotic urological surgery reduces post operative pain; the benefits of this reduces morbidity, enables a shorter hospital stay, costs less and increases patient satisfaction [1, 2]. Although robotic urological surgery reduces postoperative pain due to a reduction in tissue handling and a reduced incision size [3], no procedure is pain free and there are different challenges in treating this specific type of pain.

Pain relief is crucial but risks and side effects from the analgesic technique must also be taken into consideration and not cause increased morbidity such as nausea, ileus,

\section{KARGER}

Fax +41 613061234

E-Mailkarger@karger.ch

www.karger.com
(C) 2015 S. Karger AG, Basel

$1015-9770 / 14 / 0091-0005 \$ 38.00 / 0$

Accessible online at:

www.karger.com/cur reduction in consciousness and autonomic dysfunction thus preventing the early discharge that the robotic surgery has facilitated in the first place [1].

There are multiple analgesic techniques available to prevent and treat pain specifically caused by urological laparoscopic surgery; this article will explain the mechanism of pain pathways involved in laparoscopic procedures and review current evidence pertaining to systemic and regional analgesia methods.

\section{Causes and Mechanisms of Pain}

There are several types of pain associated with robotic surgery: incisional port site pain, pain from the peritoneum being distended with carbon dioxide, visceral pain, and shoulder tip pain. Rapid insufflation of the peritoneum with carbon dioxide causes tearing of blood vessels, traumatic traction of nerves and release of inflammatory mediators [1]. Residual gas post procedure causes shoulder tip pain, back pain and upper abdominal pain by diaphragmatic stretching and phrenic nerve irritation $[4$, 5]. The fact that there are different mechanisms of pain involved in laparoscopy makes it challenging to treat.

The most severe pain is immediately post operation $[1,6]$ and decreases with time; however there are often further peaks of pain up to 3 days later which must be effectively and safely managed in an outpatient setting. If pain is not treated effectively re-admission for pain makes the previous benefit of laparoscopic surgery for a 


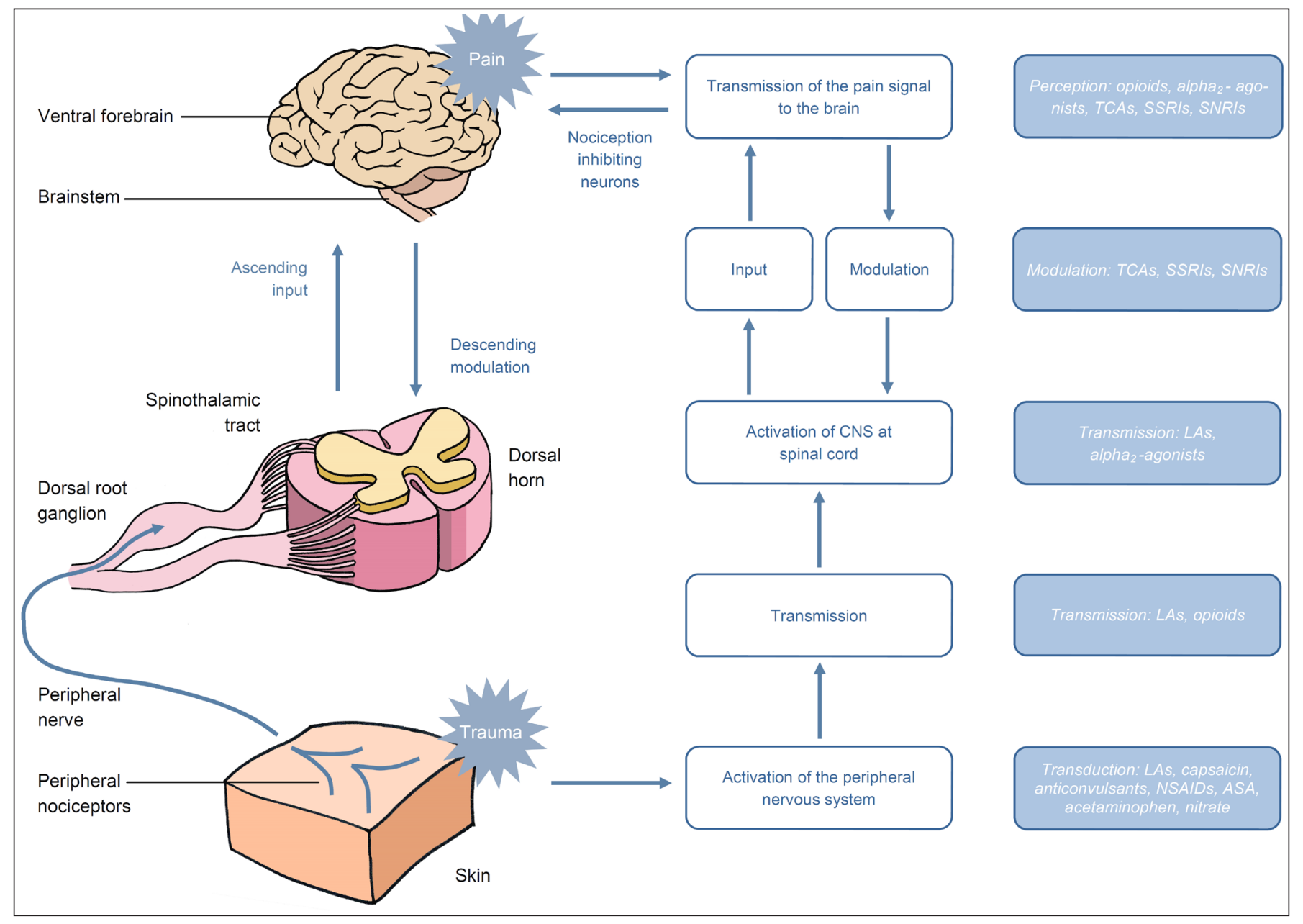

Fig. 1. Pain pathway and regions on anesthetic intervention for pain control.

shorter hospital stay redundant; conversely if strong analgesics are used inappropriately in the out patient setting this may too be problematic.

Literature searches specifically for analgesia for robotic urological surgery were carried out and yielded few results, there is lacking evidence for techniques used specifically in urological laparoscopic procedures and further studies are needed in this area. However, searches for laparoscopic procedures not specific to urological surgery can be extrapolated and used to mount evidence for best practice and therefore have been included for discussion.

\section{Methods}

Literature searches were carried out using Medline (PubMed), Cochrane, and Tripdata. Search words specifically for urological procedures are urology + analgesia + pain + robotic + laparos- copy. This yielded 98 results of which studies were excluded for not having a type/method of analgesia for robotic/laparoscopic surgery as a primary reason for the study. This resulted in only five studies comparing different analgesic methods which have been reviewed first in this study.

The urological laparoscopic studies based their research on robotic/laparoscopic general surgery and gynecological procedures, therefore, due to lack of evidence from the preliminary search a second search was carried out. Search words included the analgesia/technique+ laparoscopy + surgery + analgesia.

\section{Review of Analgesia for Robotic Urological Procedures}

The first literature review was specific to analgesia for urological procedures. Four of the 5 were retrospective studies and only one was a prospective double-blind study. All had very small numbers of participants with a total of 494 participants and 300 of those coming from 
one trial, the remainder were split amongst 4 very small trials. Two of the studies looked at the use of the local anesthetic bupivacaine, two looked at ketorolac as an adjunct to analgesia, and one looked at multimodal analgesia using NSAIDs and pregabalin. Various urological procedures were involved in the study including prostatectomy, nephrectomy and pyeloplasty, however all were laparoscopic. End points were reduction in pain scores, reduction in analgesic requirements-especially opioid based, reduction in length of stay, incidence of nausea and vomiting, and serum creatinine levels.

The first small retrospective trial by Trabulsi et al. [7] looked at multimodal analgesia in 60 patients who were undergoing robotic-assisted laparoscopic radical prostatectomy. In Group I, 30 patients received pre-emptive pregabalin, calecoxib and $975 \mathrm{mg}$ paracetamol 2 hours prior to surgery. In Group II, 30 patients received post operative intravenous ketorolac, oxycodone and $325 \mathrm{mg}$ paracetamol. Mean morphine administration was measure intra- and post-operatively. The study showed a reduction of mean total morphine administration in Group I ( $p<0.001)$ but no changes to length of stay, antiemetic use or post-operative serum creatinine levels. There are multiple variables and confounding factors with this study (different timings of medications, different paracetamol doses, different NSAIDs used, and no mention of regional anesthesia or local infiltration).

Yoost et al. [8] investigated how continuous infiltration of bupivacaine into the incision site via an elastomeric pump reduced pain post operatively of patients undergoing laparoscopic nephrectomy and compared it with intravenous and oral analgesia. Thirty-eight patients were included in the study of which 18 had local anesthetic infiltration and 20 had systemic analgesia. They stated that initial experience suggested a reduction in opioid use and a reduction in length of stay compared to those not receiving local anesthetic infiltration. However, this again is a very small retrospective study which did not give statistically significant results.

Another study involving bupivacaine looked at the effectiveness of aerosolized intraperitoneal bupivacaine to reduce post operative pain [9]. This study was done in the pediatric population undergoing laparoscopic pyeloplasty. Forty-one cases were reviewed in this retrospective trial, all received 'standard' peri-operative analgesia (ketorolac at the end of surgery, plus codeine/morphine/ hydrocodone or oxycodone prn). Five cases also received intraperitoneal aerosolized bupivacaine just prior to trochar removal and 17 had aerosolized bupivacaine earlier in the procedure (prior to incising peri-renal fascia).

Post-Operative Pain Management in Robotic Urological Surgery
Nineteen children had no bupivacaine intervention. The study showed a reduction in post-operative opioid use in the group that had bupivacaine at the beginning of surgery but not in those who received it at the end of the procedure. All 22 patients receiving some form of bupivacaine had shorter length of stay $(\mathrm{p}<0.01)$. This is a small retrospective study in just a pediatric population however it demonstrates some benefit of pre-emptive multimodal analgesia in laparoscopic urological procedures.

The largest retrospective study by Breda et al. [10] found involved 300 patients undergoing laparoscopic donor nephrectomy. In non-urological studies ketorolac has been proven to reduce opioid requirement by $50 \%$ and owing to opioids propensity to cause nausea and vomiting and constipation the authors were keen to promote its use to improve patient experience and to shorten length of stay, however, they were concerned about the effect ketorolac may have on renal function. All subjects received ketorolac (first does given on removal of the kidney, then every 6 hours for a maximum of 48 hours) as well as a strict bowel preparation regime pre-operatively. The results showed mean hospital stay of 1.1 days $(96.7 \%$ discharged on day 1), $97 \%$ ate post-operatively with no nausea/vomiting and $97 \%$ of patients received only oral analgesia. Only 6 patients required additional post-operative intravenous opioid analgesia. It is not clear how much bowel preparation and ketorolac contributed individually to the results and so it would be useful to investigate this separately, there also seems to be significant bias in this study. It does however give strength to the analgesic effect of ketorolac as adjunct analgesia and in the selected patients did not cause renal impairment.

The only prospective trial by Chow et al. [11] was a double blind study looking at the effect of ketorolac administration after laparoscopic urological surgery. Fifty-five subjects completed the study and all received patient controlled analgesia post operatively. Half the subjects also received ketorolac whilst half received a placebo and underwent a variety of urological procedures. Analogue pain scores were used to measure pain; average pain score was lower in those receiving ketorolac $(\mathrm{p}<0.005)$ and mean morphine use was less in the ketorolac group $\mathrm{p}=0.077$. There was no demonstrated change in the length of stay, blood loss, time to resume oral intake, or creatinine levels between the two groups. It was noted that patients undergoing laparoscopic pyeloplasty needed more opioid analgesia than other procedures. In this small study ketorolac was found to decrease subjective perception of pain and decrease opioid use.

Curr Urol 2015;9:5-11 
Robotic urological laparoscopic procedures are relatively new and for this reason there is a paucity in data in the most suitable analgesia for these procedures. The above studies are too few in number and lack power; they are also too heterogenous to enable statistical analysis. There is a need for good quality highly powered randomized controlled trials. Due to this, we have to base our preliminary research on laparoscopic studies in colorectal and gynecological surgery.

Taking the above into consideration a second literature search was carried out looking at specific techniques which are already used for analgesia in other laparoscopic procedures and could prove efficacious for urological procedures and provide a basis for future investigation.

\section{Practical Solutions}

There is correlation of amount of residual intra-peritoneal gas and pain scores post operatively. Therefore using lower abdominal pressures when insufflating and aspirating residual gas at the end of the procedure reduce post operative pain [4]. A Cochrane database systematic review of 15 trials (690 patients) also demonstrated less shoulder tip pain and reduced analgesic requirement with lower pressure pneumoperitoneum. A randomized controlled trial by Yasir et al. [13] compared low pressure $(8 \mathrm{mmHg})$ and high pressure $(14 \mathrm{mmHg})$ for abdominal insufflation for laparoscopic cholecystectomy; this study demonstrated reduced reporting of shoulder tip pain (reduced frequency and intensity) 4 hours post operatively though did not show statistical significance at 8 and 24 hours. Analgesic requirement and shorter hospital stay were also shown amongst the lower pressure group.

Another study by Sandhu et al. [14] showed a tendency for less pain but in this trial of 140 patients this reduction was not statistically significant. Mechanical ventilation tidal volumes did not have any effect on shoulder tip pain post laparoscopic surgery in one prospective trial containing 64 patients [15].

\section{Multimodal}

Multimodal analgesia involves the use of different classes of analgesics and different sites of analgesic administration to provide superior dynamic pain relief with reduced opioid analgesia-related side effects [16]; this can be particularly challenging in laparoscopic procedures due to the different types of pain produced by the procedure [1].
Those undergoing multimodal analgesia have shown improved recovery, less nausea and vomiting, and less opiate side effects; these can culminate into shorter hospital stays, less morbidity and increased patient satisfaction.

Multi-modal analgesia should utilize non-opiate systemic analgesics plus regional techniques, these should be administered/performed pre-emptively [17].

\section{Pre-emptive}

The initial concept of preemptive analgesia was formulated by Crile [18] in 1913 when he described the use of regional techniques to prevent post operative pain; it is thought to prevent central sensitization and hyper-excitability which decreases post operative pain by preventing wind-up and is thought to decrease the incidence of chronic pain [19]. Pre-emptive analgesia is defined as any treatment that prevents establishment of central sensitization caused by incisional and inflammatory injuries and should start before incision, cover the surgical period and the initial post operative period [10, 19, 20].

There remains controversy over the effectiveness and timing of preemptive analgesia, there is only one study which looks at preemptive analgesia in a urological laparoscopic procedures and one systematic review and meta-analysis from non-urological studies that looks at the impact of local analgesia timing and postoperative pain. Coughlin et al. [21] analyzed 26 studies and showed that surgeons should use local analgesia in laparoscopic surgery to decrease postoperative pain (infiltration at port sites or intraperitoneally), but the timing of administration is significant only for intraperitoneal infiltration but not for port infiltration with local anesthetic. Pre-incisional use of bupivacaine has been recommended (Grade A evidence) in another systematic review of interventions in laparoscopic cholecystectomy [22].

\section{Systemic Analgesia}

There were no reviews of preemptive systemic analgesia although a review of 8 prospective, double blind, randomized controlled trials mostly showed benefit in using adjunct systemic analgesia in laparoscopy prior to incision [7, 23-29]. Ketamine showed a reduction in pain in one trial but made no difference in pain relief in another, however, the overall conclusion in 8 of the trials (746 participants) showed statistically significant results 
in the reduction of pain. Drugs used involved NSAIDs (diclofenac, paracoxib, etorcoxib, calecoxib), ketamine, gabapentin and intravenous lignocaine. NSAIDs and cox-2 inhibitors showed promising results and showed benefit from their administration and demonstrated a good side effect profile.

Paracetamol has been shown to be effective and should be used in combination with other analgesics but can not be used in isolation [30]. Although these randomized controlled trials are promising, there is a need for more good quality randomized controlled trials in laparoscopic procedures plus systematic review and meta-analysis of these studies and then application to urological procedures.

Intravenous lidocaine infusion has shown benefit in analgesia in patients undergoing laparoscopic and open procedures. Although no data regarding urological procedures, it has been described in laparoscopic cholecystectomy and colorectal surgery. In a systematic review of 764 patients, 369 patients received intravenous lidocaine and underwent an open or laparoscopic procedure [31]. The results showed in both the open and laparoscopic groups a reduction in pain, reduced opioid consumption, earlier return of bowel function and shorter hospital stays. The study also states that there was no toxicity or clinically significant adverse effects. Lidocaine seems to be a safe, effective, and economical and therefore warrants further investigation as an intravenous analgesic [32].

Magnesium has also been used and compared to lidocaine as an analgesic in laparoscopic cholecystectomy. In a small (120 subject 40 in each group) double blinded study magnesium and lidocaine both reduced intra operative and post operative opioid requirements and pain scores [33]. Magnesium showed a reduction in pain scores and opioid sparing effect in another randomized controlled trial [34] though further research is required. The use of magnesium gives us another potential target for non-opioid analgesia in laparoscopic surgery.

Clonidine has been used intrathecally in spinal anesthesia pain and orally as a premedication prior to laparoscopic cholecystectomy [35]. No statistical difference was found the oral clonidine premedication group but this was an extremely small study that would need to be done on a larger scale to increase its power. In a meta-analysis intrathecal clonidine extends the time to first analgesia and decreases the amount of morphine used. However, there was also an increase in hypotension which must be considered as well as the high risk:benefit of spinal anesthesia in laparoscopic procedures [36].

Post-Operative Pain Management in Robotic Urological Surgery
Steroids (dexamethasone and methyl prednisolone) have been shown to reduce post operative nausea and vomiting and post operative pain relief, both of which will improve patient well being and aid earlier discharge. In a systematic review of 1,801 women undergoing laparoscopic gynecological procedures prophylactic dexamethasone decreased nausea and vomiting but did not show a difference in analgesic requirements [37]. Conversely in another met-analysis on colorectal laparoscopic procedures by Joshi et al. [38] showed that preemptive dexamethasone improved post operative pain relief as well as nausea and vomiting. They also recommended infiltration of surgical incisions with local anesthetic, NSAIDs, paracetamol and rescue opioids. Intravenous lignocaine was recommended as a second line treatment and neuroaxial blocks were discouraged.

\section{Regional Analgesia}

\section{Neuro Axial Blockade}

Epidural anesthesia lacked superiority over other multimodal analgesic techniques in one evidence based review of laparoscopic colorectal surgery undertaken by the procedure-specific postoperative pain management (PROSPECT) working group [38]. Minimally invasive procedures are less painful and there are more adverse effects associated with epidural analgesia (hypotension, pruritis, from motor blockade [39]).They concluded that neuroaxial blocks are not necessary in colorectal laparoscopic surgery based on a high risk:benefit ratio. The same review also looked at spinal morphine versus intravenous patient controlled analgesia and concluded that although there are reduced pain scores with intrathecal opiates there is a higher incidence of pruritis and respiratory depression. The risk:benefit ratios need to be carefully considered taking into account using neuroaxial blockade for urological laparoscopic procedures although there may be benefit if there is a high probability of converting to an open procedure.

\section{Intercostal Block}

The somatic supply of the skin, muscles and parietal peritoneum of the anterior abdominal wall are from the anterior divisions of T7-L1. T7 to T11 are intercostal nerves so intercostal blocks may be of benefit in pain relief caused by laparoscopic surgery in the upper abdomen; intercostal nerves 9,10 and 11 are likely to be blocked from a transversus abdominis plane (TAP) block

Curr Urol 2015;9:5-11 
therefore extending this block by use of bilateral intercostal blocks may be of benefit in laparoscopic procedures.

One prospective, double-blinded randomized controlled trial involving 61 patients undergoing laparoscopic cholecystectomy reported that patients who had received intercostal block had a significantly lower pain score $(\mathrm{p}<0.001)$ than those who did not receive an intercostal block [40].

\section{TAP Block}

A Cochrane review looked at the benefit of TAP blocks for non-laparoscopic procedures; 8 studies with 358 participants showed limited evidence of their benefit (analgesia and reduction in opioid use) however further studies were awaited and will be reviewed again in the future [41]. Another review and meta-analysis of the clinical effectiveness of TAP blocks was published by Johns et al. [42]; the authors concluded that the block could be used as part of multimodal analgesia and enhanced recovery. It was safe and reduced opioid requirements. A review of TAP blocks for laparoscopic procedures and the methods used to carry out TAP blocks (blind, ultrasound guided or laparoscopic guided) is required; there is also a need for comparison with other regional techniques.

\section{Paravertebral Block (PVB)}

The use of this block was used in an expanded case report of 30 patients undergoing hand-assisted laparoscopic nephrectomy and compared with conventional opioid therapy [43]. They found that utilizing a PVB as part of multi-modal analgesia improved dynamic pain scores and decreased opioid requirements. A prospective randomized controlled trial was carried out in patients who had a PVB (level T5-T6) undergoing laparoscopic cholecystectomy [44]; the small study group failed to show a statistically significant decrease in post operative pain. Larger randomized controlled trial are lacking and due to a skill shortage in Anesthetists trained to carry out this block this will be difficult to accomplish.
Autonomic nervous system may be involved in the pathogenesis of acute as well as chronic pain [45] and Stellate ganglion blockade may contribute to post operative pain relief [46].

\section{Conclusion}

In our experience caudal analgesia (with bupivacaine $0.25 \% 40 \mathrm{ml}$, clonidine $150 \mu \mathrm{g}$ and fentanyl $100 \mu \mathrm{g}$ ) provides very good intraoperative and post operative pain relief after robotic assisted laparoscopic prostatectomy (ongoing observational study). Although limited data there seems to be benefit from pre-emptive, multi-modal analgesia, use of systemic analgesic adjuncts and regional anesthesia. There is less benefit and also undesirable side effects from large quantities of systemic opioids including those delivered by patient controlled analgesia systems and neuroaxial blockade.

Robotic urological procedures are relatively new but offer great benefit to patients-reduction in morbidity, shorter hospital stay, economical benefit and increased patient satisfaction. However, pain from laparoscopic procedures can prove difficult to treat and will have a negative impact if not dealt with effectively and with minimal side effects.

The current urological laparoscopic studies are limited by lack of good quality randomized controlled prospective trials and small participant number. There is huge scope for further study and research in this area especially since more urological robotic laparoscopic surgery is being successfully carried out.

\section{Acknowledgement}

Mr Matthew Fowler for drawing figure 1.

\section{References}

1 Alexander JI: Pain after laparoscopy. Br J Anesth 1997;79:369-378.

2 Recart A, Duchene D, White PF, Thomas T, Johnson DB, Cadeddu JA: Efficacy and safety of fast-track recovery strategy for patients undergoing laparoscopic nephrectomy. J Endourol 2005;19:1165-1169.
3 Midgley S, Tolley DA: Anaesthesia for laparoscopic surgery in urology. EAU-EBU Update Series 2006;4:241-245.

4 Fredman B, Jedeikin R, Olfsanger D, Flor P, Gruzman A: Residual pneumoperitoneum: a cause of postoperative pain after laparoscopic cholecystectomy. Anesth Analg 1994;79: $152-154$.
5 Jackson SA, Laurence AS, Hill JC: Does post-laparoscopy pain relate to residual carbon dioxide? Anaesthesia 1996;51:485-487.

6 Ekstein P, Szold A, Sagie B, Werbin N, Klausner JM, Weinbroum AA: Laparoscopic surgery may be associated with severe pain and high analgesia requirements in the immediate postoperative period. Ann Surg 2006; 243:41-46. 
7 Trabulsi EJ, Patel J, Viscusi ER, Gomella LG, Lallas CD: Preemptive multimodal pain regimen reduces opioid analgesia for patients undergoing robotic-assisted laparoscopic radical prostatectomy. Urology 2010;76: 1122-1124.

8 Yoost TR, McIntyre M, Savage SJ: Continous Infusion of local anesthetic decreases narcotic use and length of hospitalisation after laparoscopic renal surgery. J Endourol 2009; 23:632-636.

9 Freilich DA, Houck CS, Meier PM, Passerotti CC, Retik AB, Nguyen HT: The effectiveness of aerosolized intraperitoneal bupivacaine in reducing postoperative pain in children undergoing robotic-assisted laparoscopic pyeloplasty. J Pediatr Urol 2008;4:337-340.

10 Breda A, Bui MH, Liao JC, Schulam PG: Association of bowel rest and ketorolac analgesia with short hospital stay after laparoscopic donor nephrectomy. Urology 2007;69:828831.

11 Chow GK, Fabrizio MD, Steer T, Potter SR, Jarrett TW, Gelman S, Kavoussi LR: Prospective double-blind study of effect of ketorolac administration after laparoscopic urological surgery. J Endourol 2001;15:171-174.

12 Gurusamy KS, Samraj K, Davidson BR: Low pressure versus standard pressure pneumoperitoneum in laparoscopic cholecystectomy. Cochrane Database Syst Rev 2009;2: CD006930.

13 Yasir M, Mehta KS, Hussain V, Banday, Aiman A, Masood I, Iqbal B: Evaluation of post operative shoulder tip pain in low pressure versus standard pressure pneumoperitoneum during laparoscopic cholecystectomy. Surgeon 2012;10:71-74.

14 Sandhu T, Paiboonworachat S, Ko-iam W: Effects of preemptive analgesia in laparoscopic cholecystectomy: a double-blind randomized controlled trial. Surg Endosc 2011;25:23-27.

15 Shin HY, Kim SH, Lee YJ, Kim DK: The effect of mechanical ventilation tidal volume during pneumoperitoneum on shoulder pain after a laparoscopic appendectomy. Surg Endosc 2010;24:2002-2007.

16 Joshi GP: Multimodal analgesic techniques and postoperative rehabilitation. Anesthesiol Clin North America 2005;23:185-202.

17 Ahn Y, Woods J, Connor S: A systematic review of interventions to facilitate ambulatory laparoscopic cholecystectomy. HPB (Oxford) 2011;13:677-686.

18 Crile GW: The kinetic theory of shock and its prevention through anoci-association. Lancet 1913;185:7-16

19 Kissin I: Preemptive analgesia. Anesthesiology 2000;93:1138-1143.

20 Dahl JB, Møiniche S: Pre-emptive analgesia. Br Med Bull 2004;71:13-27.

21 Coughlin SM, Karanicolas PJ, Emmerton-Coughlin HM, Kanbur B, Kanbur S, Colquoun PH: Better late than never? Impact of local analgesia timing on postoperative pain in laparoscopic surgery: a systematic review and metaanalysis. Surg Endosc 2010;24: $3167-3176$
22 Pourseidi B, Khorram-Manesh A: Effect of intercostals neural blockade with Marcain (bupivacaine) on postoperatove pain after laparoscopic cholecystectomy. Surg Endosc 2007;21:1557-1559.

23 Kim TH, Kang H, Choi YS, Park JM, Chi $\mathrm{KC}$, Shin HY, Hong JH: Pre- and intraoperative lidocaine injection for preemptive analgesics in laparoscopic gastrectomy: a prospective, randomized, double-blind, placebo-controlled study. J Laparoendosc Adv Surg Tech A 2013;23:663-668.

24 Singh H, Kundra S, Singh RM, Grewal A, Kaul TK, Sood D: Preemptive analgesia with ketamine for laparoscopic cholecystectomy. J Anaesthesiol Clin Pharmacol 2013;29:478484.

25 Bunyavejchevin S, Prayoonwech C, Sriprajittichai P: Preemptive analgesic efficacy of parecoxib vs placebo in infertile women undergoing diagnostic laparoscopy: randomized controlled trial. J Minim Invasive Gynecol 2012;19:585-588.

26 Ratchanon S, Phaloprakarn C, Traipak K: Pain control in laparoscopic gynecologic surgery with/without preoperative (preemptive) parecoxib sodium injection: a randomized study. J Med Assoc Thai 2011;94:1164-1168.

27 Wang Q, Li Z, Wang ZP, Cui C: Preemptive analgesic effect of parecoxib sodium in patients undergoing laparoscopic colorectal surgery. Nan Fang Yi Ke Da Xue Xue Bao 2010; 30:2556-2557.

28 Nesek-Adam V, Grizelj-Stoj i E, Mrši V, Raši Z, Schwarz D: Preemptive use of diclofenac in combination with ketamine in patients undergoing laparoscopic cholecystectomy: a randomized, double-blind, placebo-controlled study. Surg Laparosc Endosc Percutan Tech 2012;22:232-238.

29 Phinchantra P, Bunyavehchevin S, Suwajanakorn S, Wisawasukmongchol W: The preemptive analgesic effect of celecoxib for day-case diagnostic laparoscopy. J Med Assoc Thai 2004:87:283-288.

30 Gousheh SM, Nesioonpour S, Javaher Foroosh F, Akhondzadeh R, Sahafi SA, Alizadeh Z: Intravenous paracetamol for postoperative analgesia in laparoscopic cholecystectomy. Anesth Pain Med 2013;3:214-218.

31 McCarthy GC, Megalla SA, Habib AS: Impact of lidocaine infusion on postoperative analgesia and recovery from surgery: a systematic review of randomised controlled trials. Drugs 2010;70:1149-1163.

32 De Oliveira GS Jr, Fitzgerald P, Streicher LF, Marcus RJ, McCarthy RJ: Systemic lidocaine to improve postoperative quality of recovery after ambulatory laparoscopic surgery. Anesth Analg 2012;115:262-267.

33 Saadawy IM, Kaki AM, Abd El Latif AA, Abd-Elmaksoud AM, Tolba OM: Lidocaine vs magnesium: effect on analgesia after laparoscopic cholecystectomy. Acta Anaesthesiol Scand 2010;53:549-556.
34 Mentes O, Harlak A, Yigit T, Balkan A, Balkan M, Cosar A, Savaser A, Kozak O, Tufan $\mathrm{T}$ : Effect of intraoperative magnesium sulphate infusion on pain relief after laparoscopic cholecystectomy. Acta Anaesthesiol Scand 2008;52:1353-1359.

35 Sung CS, Lin SH, Chan KH, Chang WK, Chow LH, Lee TY: Effect of oral clonidine premedication on perioperative hemodynamic response and postoperative analgesic requirments for patients undergoing laparoscopic cholecystectmy. Acta Anaesthesiol Sin 2000;38:23-29.

36 Engelman E, Marsala C: Efficacy of adding clonidine to intrathecal morphine in acute postoperative pain: meta-analysis. Br J Anaes 2013;110:21-27.

37 Wang B, He KH, Jiang MB, Liu C, Min S: Effect of prophylatic dexamethasone on nausea and vomiting after laparoscopic gynacological operation: meta-analysis. Middle East J Anesthesiol 2011;21:397-402.

38 Joshi GP, Bonnet F, Kehlet H: Evidence-based postoperative pain management after laparoscopic colorectal surgery. Colorectal Dis 2013;15:146-155.

39 Wheatley RG, Schug SA, Watson D: Safety and efficacy of postoperative epidural analgesia. Br J Anaesth 2001;87:47-61.

40 Pourseidi B, Khorram-Manesh A: Effect of intercostals neural blockade with Marcaine (bupivacaine) on postoperative pain after laparoscopic cholecystectomy. Surg Endosc 2007;21:1557-1559.

41 Charlton S, Cyna AM, Middleton P, Griffiths JD: Perioperative transversus abdominis plane (TAP) blocks for analgesia after abdominal surgery. Cochrane Database Syst Rev 2010;(12):CD007705.

42 Johns N, O'Neill S, Ventham NT, Barron F, Brady RR, Daniel T: Clinical effectiveness of transversus abdominis plane (TAP) block in abdominal surgery: a systematic review and meta-analysis. Colorectal Dis 2012;14:e635642

43 Clendenen SR, Wehle MJ, Rodriguez GA, Greengrass RA: Paravertebral block provides significant opioid sparing after hand-assisted laparoscopic nephrectomy: an expanded case report of 30 patients. J Endourol 2009;23: 1979-1983.

44 Naja MZ, Ziade MF, Lonnqvist PA: General Anaesthesia combined with bilateral paravertebral blockade (T5-T6) vs general anaesthesia for laparoscopic cholecystectomy: a prospective randomised clinical trial. Eur J Anaesthesiol 2004;21:489-495.

45 Bantel C, Trapp S: The role of the autonomic nervous system in acute surgical pain processing - what do we know? Anaesthesia 2011;66:541-544.

46 McDonnell JG, Finnerty O, Laffey JG: Stellate ganglion blockade for analgesia following upper limb surgery. Anaesthesia 2011;66: 611-614. 